\title{
Estimation of some exponential sums containing the fractional part function and some other "non-standard" exponential sums
}

\author{
by \\ Grigori KolesniK (Los Angeles, CA)
}

1. Introduction. Some problems in number theory and some other branches of mathematics can be reduced to the estimation of exponential sums

$$
\sum_{X_{1}<x \leq X_{2}} e(F(x)) \quad \text { with } X=X_{2}-X_{1} \leq X_{1} .
$$

If $F(x)$ is a polynomial or a function which can be reduced to a polynomial then the sum can be evaluated by using Vinogradov's method; if $F(x)$ is "van der Corput" type function then one uses van der Corput's method or Bombieri-Iwaniec method. Here by van der Corput (v.d.c.) type function of order $k$ we mean a real-valued $k$ times continuously differentiable function $F(x)$ such that $F^{(j)}(x) \asymp F_{j}(x) / x^{j}(j=1, \ldots, k)$ with piecewise monotone $F_{j}(x)$ such that if $k>1$, then

$$
1 \lll F_{j+1}(x) / F_{j}(x) \ll 1 \text { and } \varlimsup x^{1-2 / K} F^{(k)}(x) \ll 1 ;
$$

if $k=1$, then

$$
\lim _{x \rightarrow \infty} F_{1}(x)=\infty \quad \text { and } \quad \varlimsup\left|F^{\prime}(x)\right|<1
$$

(see the notation below).

Note that if $k>1$ is the smallest integer such that $F(x)$ is a v.d.c. function of order $k$ and $K=2^{k}$ then

$$
F^{(k)}(x) \lll x^{2 / K-1} \text { and } F^{(k-1)}(x) \ggg x^{4 / K-1}
$$

so that

$$
x^{4 / K-2} \lll F^{(k)}(x) \lll x^{2 / K-1} .
$$

If $X$ is "not small", the above mentioned methods give non-trivial estimates. We call such sums standard exponential sums. If $X$ is "small", the

2000 Mathematics Subject Classification: Primary 11L03. 
sum is called short and the well-known van der Corput's estimates may be larger than the trivial estimates. Also, if $F(x)$ contains an oscillating term, van der Corput's method cannot be used directly. We call such sums non-standard exponential sums. In the past we studied short sums [2] and sums containing an oscillating term [1], [2].

Wenguang Zhai has recently introduced [4] a method of evaluation of exponential sums with $F(x)=f(x)+g(x)\{h(x)\}$. He applied the method to prove that for any $k \neq 0$ and any $c>0$ the sequence $\left\{\left[n^{c}\right] \log ^{k} n\right\}$ is uniformly distributed modulo 1 by proving that the discrepancy of the sequence satisfies

$$
D(X) \ll X^{-\delta(c)} \log X \quad \text { for some } \delta(c)>0 .
$$

His result improved the result of Rieger [3] who proved the uniform distribution of the sequence for $1<c<3 / 2$ and $0<k<1$.

The method of Zhai gives a non-trivial estimate if $f(x), g(x)$ and $h(x)$ are v.d.c. functions and $g(x) \ll x^{3 / 4-\alpha}$ for any fixed $\alpha>0$. One can evaluate such sums (and more general sums) with $g(x) \ll x^{1-\alpha}$ using our method of evaluation of short sums and

Lemma 1. Let $f(t, x)$ be a real-valued function such that

$$
\left|f\left(t_{1}, x\right)-f\left(t_{2}, x\right)\right| \leq \lambda\left|t_{1}-t_{2}\right| .
$$

Then for any real function $g(x)$, any positive integer $r$ and any $M>0$ we have

$$
\begin{aligned}
S= & \sum_{x} a(x) e(f(g(x),\{h(x)\})) \\
= & \frac{1}{M} \sum_{m=0}^{M-1} \sum_{j=0}^{\infty} b_{j, m} \sum_{x} a(x) e(f(g(x), m / M)+j h(x)) \\
& +O\left(\frac{\lambda r+r}{M} \sum_{x}|a(x)|\right) \\
& +O\left(\frac{r}{M} \sum_{j=0}^{\infty} \frac{\sin (2 \pi r j / M)}{\sin (\pi j / M)} a_{j} \sum_{x}|a(x)| e(j h(x))\right),
\end{aligned}
$$

where

$$
\begin{aligned}
a_{j} & =(\sin (\pi j / M) /(\pi j / M))^{r+1}, \quad a_{0}=1, \\
b_{j, m} & =a_{j} e(-(2 m+1) j /(2 M)) .
\end{aligned}
$$

This lemma is also simpler to use than the corresponding lemma of Zhai. Using Lemma 1, we prove

THEOREM 1. Let $k$ be a sufficiently large positive integer such that $f(x)$, $g(x)$ and $h(x)$ are v.d.c. functions of order $k$ and let $k_{1} \in[2, k-1]$ and 
$k_{2} \in[2, k-2]$ be the smallest integers such that $f(x), g(x)$ and $h(x)$ are v.d.c. functions of orders $k_{1}, 1$ and $k_{2}$ respectively. Assume that $g(x) \ll x^{1-\alpha}$ for some $\alpha>0$ and that for any $m$ the functions $f_{m}(x) / h_{m}(x)$ are piecewise monotone on $\ll 1$ intervals and

$$
\left|f_{m}(x) h_{m+1}(x) /\left(f_{m+1}(x) h_{m}(x)\right)-1\right| \ggg 1 .
$$

Define

$$
\varphi_{j}(y)=f^{(j)}\left(g^{-1}(y)\right), \quad \phi_{j}(y)=h^{(j)}\left(g^{-1}(y)\right)
$$

and assume that for any $m$ the functions $\varphi_{j}^{(m)}(y) / \phi_{j}^{(m)}(y)$ are piecewise monotone on $\ll 1$ intervals and

$$
\begin{gathered}
\left|\varphi_{j}^{(m)}(y) \phi_{j}^{(m+1)}(y) /\left(\varphi_{j}^{(m+1)}(y) \phi_{j}^{(m)}(y)\right)-1\right| \ggg 1, \\
\left|\varphi_{1}^{(p)}(y)\right| \ll y^{2 / P-3}, \quad\left|\phi_{1}^{(p)}(y)\right|<y^{2 / P-5} \quad \text { for some integer } p>1 .
\end{gathered}
$$

Then

$$
S \equiv \sum_{X \leq x \leq 2 X} e(f(x)+g(x)\{h(x)\}) \ll X \Delta_{0}
$$

where

$$
\Delta_{0}=X^{-\alpha /(3 P)}+\left(G+X^{1 / 3}\right)^{-1 /(P K)}, \quad G=g(X) .
$$

Also, if $f(x)=C h(x)$ then the above estimate holds if $|C|>1$ and

$$
|S| \ll X \Delta_{0}+X / G \quad \text { if }|C|<1 .
$$

THEOREM 2. Let $f(x, y)$ be a real-valued function on $[X, 2 X] \times[0,1]$ such that for any $y$ it is a v.d.c. function of order $k$. Assume that $k$ is the smallest such integer. Assume also that $g(x)$ is a v.d.c. function of order 1 such that for some $a>0$ we have $g(x) \ll x^{1-a}$ and, setting $h(n)=f\left(g^{-1}(n), n\right)$, assume that it is a v.d.c. function of order $j$. Let $\lambda_{k}$ and $\mu_{j}$ be such that

$$
\left|\partial^{k} f(x, y) / \partial x^{k}\right| \asymp \lambda_{k} \text { and }\left|h^{(j)}(n)\right| \asymp \mu_{j} .
$$

Then

$$
\begin{aligned}
S & \equiv \sum_{X \leq x \leq 2 X} e(f(x,\{g(x)\})) \\
& \ll X\left[\lambda_{k}^{1 /(K-2)}+X^{-a / K}+G(X)^{-2 / K}+\mu_{j}^{4 /(K J+K)}\right] .
\end{aligned}
$$

For the sequence $\left\{\left[n^{\alpha}\right] \log ^{\beta} n\right\}$ considered by Rieger and Zhai,

$$
f(x)=x^{\alpha} \log ^{\beta} x, \quad g(x)=-\log ^{\beta} x, \quad h(x)=x^{\alpha},
$$

so that if $\alpha \beta \neq 0$ the conditions of Theorem 1 are satisfied and one can use it to prove the uniform distribution of the sequence modulo 1 and to evaluate the discrepancy. One can do the same for $f(x)=x^{\alpha}, g(x)=x^{\beta}$ and $h(x)=x^{\gamma}$ with $\alpha \neq \gamma$ and $\beta<1$, and some other functions. 
2. Notation. We will use the following notation: $e(x)=\exp (2 \pi i x)$; $f(x) \ll g(x)$ means that $f(x)=O(g(x)) ; f(x) \lll g(x)$ means that $f(x) \ll$ $g(x) x^{\varepsilon} ; f(x) \asymp g(x)$ means that $f(x) \ll g(x) \ll f(x) ;\{x\},[x]$ and $\|x\|$ are the fractional part, the integer part and the distance to the nearest integer functions; $|S|$ is the cardinality of the set $S$. For positive integers $k, r$ etc., we write $K=2^{k}, R=2^{r}$ etc.

3. Proofs. To prove Lemma 1 , we take

$$
\begin{aligned}
\chi_{r, m}(x) & \equiv \chi_{r, m}(x ; \delta) \\
& =(2 \delta)^{-r} \int_{-\delta}^{\delta} \ldots \int_{-\delta}^{\delta} \chi_{0, m}\left(x+t_{1}+\ldots+t_{r}\right) d \underline{t} \quad(m=0, \ldots, M-1),
\end{aligned}
$$

where $\chi_{0, m}(x)$ is the characteristic function of $[m / M,(m+1) / M)$ modulo 1 . Expanding $\chi_{0, m}(x)$ into a Fourier series, we obtain

$$
\begin{aligned}
\chi_{r, m}(x) & =(2 \delta)^{-r} \int_{-\delta}^{\delta} \ldots \int_{-\delta}^{\delta}\left(\frac{1}{M}+\sum_{|j|=1}^{\infty} a_{j, m} e\left(x+t_{1}+\ldots+t_{r}\right)\right) d \underline{t} \\
& =\frac{1}{M}+\sum_{|j|=1}^{\infty} a_{j, m}\left(\frac{\sin (2 \pi j \delta)}{2 \pi j \delta}\right)^{r} e(j x)
\end{aligned}
$$

where

$$
a_{j, m}=\frac{\sin (\pi j / M)}{\pi j} e\left(\frac{-(2 m+1) j}{2 M}\right) .
$$

We use (3) with $\delta=1 / M$ so that $a_{j, m}(\sin (2 \pi j \delta) /(2 \pi j \delta))^{r}=b_{j, m} / M$ from the lemma. Since $\sum_{m=0}^{M-1} \chi_{0, m}(x)=1$, we have $\sum_{m=0}^{M-1} \chi_{r, m}(x)=1$ and we obtain

$$
\begin{aligned}
S= & \sum_{x} a(x) \sum_{m=0}^{M-1} \chi_{r, m}(h(x)) e(f(g(x),\{h(x)\})) \\
= & \sum_{x} a(x) \sum_{m=0}^{M-1} \chi_{r, m}(h(x)) e(f(g(x), m / M)) \\
& +\sum_{x} a(x) \sum_{m=0}^{M-1} \chi_{r, m}(h(x))[e(f(g(x),\{h(x)\}))-e(f(g(x), m / M))] .
\end{aligned}
$$

The first sum is reduced to the first sum in (2) by using (3) with $\delta=1 / M$. To evaluate the second sum (which we denote with $S_{1}$ ), we divide it into two subsums: the first subsum, $S_{1}^{\prime}$, is over all $m$ with $\|m / M\|>r / M$, and $S_{1}^{\prime \prime}$ is the remaining part of $S_{1}$. 
If $\|m / M\|>r / M$ then $\chi_{r, m}(g(x))=0$ unless $|g(x)-m / M|<r / M$. Since $|e(a)-e(b)|=2|\sin (\pi(b-a))|<2 \pi|a-b|$, we obtain

$$
S_{1}^{\prime} \ll \sum_{x}|a(x)| \sum_{m} \chi_{r, m}(h(x)) \frac{r \lambda}{M}=\frac{r \lambda}{M} \sum_{x}|a(x)| .
$$

To evaluate $S_{1}^{\prime \prime}$, we write first

$$
\left|S_{1}^{\prime \prime}\right| \leq \sum_{x}|a(x)| \sum_{m} 2 \chi_{r, m}(h(x)) \leq 2 \sum_{x}|a(x)| \chi_{1}(h(x) ; 1 /(2 M))
$$

where

$$
\chi_{1}(t ; \delta) \equiv(2 \delta)^{-r} \int_{-\delta}^{\delta} \ldots \int_{-\delta}^{\delta} \chi\left(t+t_{1}+\ldots+t_{r}\right) d \underline{t}
$$

and $\chi(t)$ is the characteristic function of $[-r / M, r / M)$ modulo 1. Similarly to $(3)$, we obtain

$$
\chi_{1}(t ; 1 /(2 M))=\frac{2 r}{M}+2 \sum_{|j|=1}^{\infty} \frac{\sin (2 \pi r j / M)}{\pi j}\left(\frac{\sin (\pi j / M)}{\pi j / M}\right)^{r} e(j t)
$$

so that

$$
\left|S_{1}^{\prime \prime}\right| \leq \frac{4 r}{M} \sum_{x}|a(x)|+2 \sum_{j=1}^{\infty} \frac{\sin (2 \pi r j / M)}{\sin (\pi j / M)} a_{j} e(j h(x)) .
$$

To prove the theorems, we need three more lemmas.

Lemma 2. Let $f(x) \in C^{(k+j)}\left[X_{1}, X_{2}\right]$ with $k>1, j>0$ and $1 \leq X=$ $X_{2}-X_{1} \leq X_{1}$. Assume that

$$
f^{(k)}(x) \leq \lambda_{k} \quad \text { and } \quad f^{(k+j)}(x) \asymp \lambda_{k+j} .
$$

Then

$$
\begin{aligned}
& \left|\sum_{X_{1} \leq x \leq X_{2}} e(f(x))\right| \ll X\left[\lambda_{k}^{1 /(K-2)}+\left(X^{-j-2} \lambda_{k} / \lambda_{k+j}\right)^{4 /(K(j+4))}\right. \\
& +\left(\lambda_{k+j} X^{4+j-8 / K}\right)^{-4 /(K(j+2))} .
\end{aligned}
$$

Lemma 2 is a simple generalization of van der Corput estimates (for the proof, see [1, Lemma 4.1]).

Lemma $3\left[2\right.$, Lemma 4.2]. Let $f(x) \in C^{2}\left[X_{1}, X_{2}\right]$ be such that $f^{\prime \prime}(x) \asymp \lambda_{2}$ for $X_{1} \leq x \leq X_{2}=X_{1}+X \leq 2 X_{1}$. Assume that $\left\|f^{\prime}(x)\right\| \geq X \lambda_{2}$. Then $\sum_{X_{1} \leq x \leq X_{2}} e(f(x)) \ll X \sqrt{\lambda_{2}}+1+\min \left\{X ; 1 / \sqrt{\lambda_{2}} ; 1 /\left\|f^{\prime}\left(X_{2}\right)\right\| ; 1 /\left\|f^{\prime}\left(X_{1}\right)\right\|\right\}$.

LEMma 4. Let $f(x, y)$ be a real-valued function on $\{(x, y): Y \leq y \leq$ $\left.2 Y, X_{1} \equiv X_{1}(y) \leq x \leq X_{2}(y) \equiv X_{2}\right\}$ such that $f(x, y)$ is a v.d.c. function 
of order $k$ as a function of $x$ and either $g_{1}(y) \equiv f^{(k-1)}\left(X_{1}, y\right)$ or $g_{2}(y) \equiv$ $f^{(k-1)}\left(X_{2}, y\right)$ is a v.d.c. function of order $j$. Assume that

$$
\frac{\partial^{k} f}{\partial x^{k}}(x, y) \asymp \lambda_{k} \quad \text { and } \quad g_{i}(y)^{(j)} \asymp \mu_{j} \quad \text { for a v.d.c. function } g_{i}(y) \text {. }
$$

Then

$$
\begin{aligned}
S & \equiv \sum_{Y \leq y \leq 2 Y}\left|\sum_{X_{1} \leq x \leq X_{2}} e(f(x, y))\right| \\
& \ll X Y\left(\lambda_{k}^{1 /(K-2)}+X^{-4 /(3 K)}+Y^{-2 / K}+\mu_{j}^{4 /(K J+K-8)}\right) \quad \text { if } k>1
\end{aligned}
$$

and

$$
S \ll X Y\left(\mu_{j}^{1 /(J-1)}+1 / Y+\log X / X\right) \quad \text { if } k=1 .
$$

Proof. If $k=1$, we use van der Corput's Lemma to get

$$
\begin{aligned}
S & \ll \sum_{y} \min \left\{X ; 1 /\left\|f_{y}\left(X_{1}, y\right)\right\|+1 /\left\|f_{y}\left(X_{2}, y\right)\right\|\right\} \\
& \ll \sum_{y} \min \left\{X ; 1 /\left\|f_{y}\left(X_{1}, y\right)\right\| ; 1 /\left\|f_{y}\left(X_{2}, y\right)\right\|\right\}
\end{aligned}
$$

and proceed as below. If $k=2$ then we use van der Corput's estimates (Lemma 2 with $j=0$ ) to get

$$
S \ll X \sqrt{\lambda_{2}}+1 / \sqrt{\lambda_{2}} .
$$

If $\lambda_{2} \gg X^{-4 / 3}$, the above implies $S \ll X Y \sqrt{\lambda_{2}}+Y X^{2 / 3}$.

If $X \lambda_{2} \equiv \Delta_{0} \leq X^{-1 / 3}$, we can evaluate $S$ differently. We define

$$
Y_{i}(\Delta) \equiv Y(\Delta)=\left|\left\{y \in[Y, 2 Y]:\left\|g_{i}(y)\right\| \leq \Delta\right\}\right| .
$$

Using Lemma 3, we obtain

$$
\text { (4) } S \ll X Y\left(X \lambda_{2}\right)+X Y \sqrt{\lambda_{2}}+\sum_{r} \min \left\{X ; 1 / \sqrt{\lambda_{2}} ; 1 /\left(2^{r} \Delta_{0}\right)\right\} Y\left(2^{r} \Delta_{0}\right) \text {. }
$$

Now we need to evaluate $Y(\Delta)$. If $\mu_{1}$ is small, we divide the interval $[Y, 2 Y]$ into $\ll Y \mu_{1}+1$ subintervals of length $\ll 1 / \mu_{1}$ such that $[g(y)]$ remains constant for all $y$ in a subinterval. Each of them contains $\ll \Delta / \mu_{1}+1$ integers $y$ such that $\|g(y)\|<\Delta$ so that

$$
Y(\Delta) \ll\left(Y \mu_{1}+1\right)\left(\Delta / \mu_{1}+1\right) \ll Y \Delta+Y \mu_{1}+1 .
$$

If $\mu_{1}$ is not small but $\mu_{k}$ is small for some $k>1$, we use (3) with $r=1$, $M=3 / \delta, m=0$ and $m=M-1$ to obtain

$$
Y(\Delta) \leq \min _{\delta \geq \Delta} Y(\delta)
$$


where

$$
\begin{aligned}
Y(\delta) \ll & Y \delta+\sum_{|l|=1}^{\infty} \min \left\{1 / M ; M l^{-2}\right\}\left|\sum_{y} e(l g(y))\right| \\
\ll & Y \delta+\sum_{l} \min \left\{1 / M ; M l^{-2}\right\} \\
& \times\left[Y\left(l \mu_{j}\right)^{1 /(J-2)}+Y^{1-2 / J} \log Y+Y^{1-8 / J+16 J^{-2}} \mu_{j}^{-2 / J}\right],
\end{aligned}
$$

and $Y(\Delta) \ll Y \Delta+Y \mu_{j}^{1 /(J-1)}$. We substitute this into (4) to obtain

$$
\begin{aligned}
S & \ll X Y \sqrt{\lambda_{2}}+X^{2} Y \lambda_{2}+X Y \mu_{j}^{1 /(J-1)}+X \sqrt{Y} \\
& \ll X Y \sqrt{\lambda_{2}}+X^{2 / 3} Y+X Y \mu_{j}^{1 /(J-1)}+X \sqrt{Y} .
\end{aligned}
$$

This proves the lemma for $k=2$. If $k>2$, we apply H. Weyl-van der Corput inequality $m=k-2$ times:

$$
\left|\frac{S}{X Y}\right|^{M} \ll Q^{-M / 2}+\frac{1}{Q^{M-1} X Y} \sum_{q_{1}=1}^{Q} \ldots \sum_{q_{m}=1}^{Q^{M / 2}} \sum_{y}\left|\sum_{X_{1}(\underline{q}) \leq x \leq X_{2}(\underline{q})} e\left(f_{1}(x, y)\right)\right|,
$$

where

$$
M=2^{m}, \quad Q=\min \left\{\lambda_{k}^{-1 /(2 M-1)} ; X^{2 / M} ; \mu_{j}^{-2 /(M(J+1)-2)}\right\}
$$

and

$$
f_{1}(x, y)=q_{1} \ldots q_{m} \int_{0}^{1} \ldots \int_{0}^{1} f_{x^{m}}\left(x+t_{1} q_{1}+\ldots+t_{m} q_{m}, y\right) d \underline{t} .
$$

Using (5), we obtain

$$
\begin{aligned}
& \left|\frac{S}{X Y}\right|^{M} \ll Q^{-M / 2}+\frac{1}{Q^{M-1} X Y} \\
& \quad \times \sum_{q_{1}, \ldots, q_{m}}\left(X Y \sqrt{q_{1} \ldots q_{m} \lambda_{k}}+X^{2 / 3} Y+X \sqrt{Y}+X Y\left(q_{1} \ldots q_{m} \mu_{j}\right)^{1 /(J-1)}\right) \\
& \ll Q^{-M / 2}+\sqrt{Q^{M-1} \lambda_{k}}+X^{-1 / 3}+Y^{-1 / 2}+\left(Q^{M-1} \mu_{j}\right)^{1 /(J-1)} \\
& \ll \lambda_{k}^{M /(K-2)}+X^{-1 / 3}+Y^{-1 / 2}+\mu_{j}^{4 M /(K J+K-8)} .
\end{aligned}
$$

To prove Theorem 1 , we assume first that $G \equiv g(X) \ll X^{1 /(3 K)}$. We use Lemma 1 with $r=3$ and $M=\max \left\{X^{1 /(2 K)} / G ; G X^{1 /(4 K)}\right\}$ to obtain

$$
\begin{aligned}
S \ll & \sum_{|j|=0}^{\infty}\left|a_{j}\right| \sum_{m=0}^{M-1}\left|\sum_{x} e(f(x)+g(x) m / M+j h(x))\right| \\
& +\frac{X G}{M}+\sum_{j=1}^{\infty}\left|a_{j}\right|\left|\sum_{x} e(j h(x))\right| .
\end{aligned}
$$


Lemma 2 with $k=k_{1}+1$ and $m=0$ shows that the last sum is

$$
\begin{aligned}
\ll X \sum_{j=1}^{\infty} \min \{1 / M & \left.; M^{3} j^{-4}\right\} \\
& \times\left[\left(j \lambda_{k_{1}+1}\right)^{1 /\left(2 K_{1}-2\right)}+X^{-2 / K}+\left(j \lambda_{k_{1}+1} X^{4-4 / K_{1}}\right)^{-1 / K_{1}}\right]
\end{aligned}
$$

$\ll X\left[\left(M \lambda_{k_{1}+1}\right)^{1 /\left(2 K_{1}-2\right)}+X^{-2 / K}+\left(M \lambda_{k_{1}+1} X^{4-4 / K_{1}}\right)^{-1 / K_{1}}\right] \ll X^{1-1 /(4 K)}$.

To evaluate the first sum, for a fixed $j$, we divide the interval $[X, 2 X]$ into $\ll \log X$ subintervals with $\left|f^{(p)}(x)+j h^{(p)}(x)\right| \asymp \lambda_{p} \gg\left|f^{(p)}(X)\right| X^{-\varepsilon_{1}}$ and one interval (which we denote with $I$ ) on which the last inequality does not hold, where $\varepsilon_{1}>0$ is a sufficiently small number and $p$ is the smallest integer such that

$$
\left|f^{(p)}(X)\right| X^{1-1 / P} \leq 1 \text { and }\left|j h^{(p)}(X) X^{1-1 / P}\right| \leq 1 .
$$

Obviously, $p<k$. The conditions of the theorem imply that if $x \in I$ then

$$
\left|f^{(p+1)}(x)+j h^{(p+1)}(x)\right| \ggg\left|f^{(p+1)}(X)\right| .
$$

Using Lemma 2 with $k=p$ and $m=1$ if $x \in I$ and $m=0$ otherwise, we find that the first sum is

$$
\ll \sum_{j} \min \left\{1 ; M^{2} j^{-2}\right\} X^{1-1 / K} \log X \ll X^{1-1 /(4 K)}
$$

so that

$$
S \ll X^{1-1 /(4 K)} .
$$

Now we assume that $G \gg X^{1 /(3 K)}$. We take $\varepsilon_{0}=\left(G^{\prime}\right)^{4 /(9 P)}+G^{-4 /(P K)}$ and define $a(x)=1-\chi(x)$ where $G^{\prime}=g^{\prime}(X)$ and $\chi(x)$ is the characteristic function of $\left[-\varepsilon_{0}, \varepsilon_{0}\right]$ modulo 1 . Then

$$
S=\sum_{X \leq x \leq 2 X} a(g(x)) e(f(x)+g(x)\{h(x)\})+O\left(\sum_{X \leq x \leq 2 X} \chi(g(x))\right) .
$$

The $O$-term is $\ll\left|\left\{x \in[X, 2 X]:\|g(x)\| \leq 3 \varepsilon_{0}\right\}\right|$. As above, we divide the interval $[X, 2 X]$ into $\ll X G^{\prime}+1$ subintervals of length $\ll 1 / G^{\prime}$ each such that $[g(X)]$ remains constant on each subinterval. The number of $x$ in each subinterval such that $\|g(x)\| \leq 3 \varepsilon_{0}$ is $\ll 1+\varepsilon_{0} / G^{\prime}$ so that the $O$-term is

$$
\ll\left(X G^{\prime}+1\right)\left(1+\varepsilon_{0} / G^{\prime}\right) \ll X \varepsilon_{0}+X G^{\prime} .
$$

Now we apply Lemma 1 with $r=3$ and $M=G X^{1 /(4 K)}$ to obtain

$$
\begin{aligned}
S \ll & \sum_{|j|=0}^{\infty}\left|a_{j}\right|\left|\sum_{m=0}^{M-1} \sum_{x} a(h(x)) e(f(x)+j h(x)+g(x) m / M+m j / M)\right| \\
& +X \varepsilon_{0}+X G^{\prime}+X^{1-1 /(4 K)}+\sum_{j=1}^{\infty}\left|a_{j}\right|\left|\sum_{x} e(j h(x))\right| .
\end{aligned}
$$


As above, the last sum is $\ll X^{1-1 /(4 K)}$. Now we need to evaluate the first sum. We denote it by $\Sigma$ and denote the sum over $m$ and $x$ by $S_{1}$; summing over $m$, we obtain

$$
S \ll \sum_{x} a(g(x)) e(f(x)+j h(x)) \frac{e(g(x))-1}{e((j+g(x)) / M)-1} .
$$

Let $G_{1}$ and $G_{2}$ be the minimum and maximum of $g(x)$ on $[X, 2 X]$.

Setting

$$
I(y)=\left\{x \in[X, 2 X]: y+\varepsilon_{0} \leq g(x) \leq y+1-\varepsilon_{0}\right\} \equiv\left[X_{1}(y), X(y)\right]
$$

and writing $j=u+v M$ with $|u|<M / 2$, we obtain

$$
S_{1} \ll \sum_{G_{1} \leq y \leq G_{2}}\left|\sum_{x \in I(y)} e(f(x)+j h(x)) \frac{e(g(x))-1}{e((u+g(x)) / M)-1}\right| .
$$

If $X_{1}(y) \leq x \leq X(y)-1$ then $1 /|e((u+g(x)) / M)-1| \ll M /\left(|u+y|+\varepsilon_{0}\right)$ and

$$
\left|\frac{1}{e((u+g(x)) / M)-1}-\frac{1}{e((u+g(x+1)) / M)-1}\right| \ll \frac{M G^{\prime}}{(y+u)^{2}+\varepsilon_{0}^{2}} .
$$

Abel's summation formula and the above inequalities yield

$$
\begin{aligned}
& S_{1} \ll \sum_{y}\left\{\frac{M}{|y+u|+\varepsilon_{0}}\left|\sum_{x \in I(y)} e(\psi(x))\right|\right. \\
& \left.+\frac{M G^{\prime}}{(y+u)^{2}+\varepsilon_{0}^{2}} \sum_{X_{1}(y) \leq s \leq X(y)}\left|\sum_{s \leq x \leq X(y)} e(\psi(x))\right|\right\},
\end{aligned}
$$

where $\psi(x)=f(x)+j h(x)+i g(x)$ and $i=0$ or 1 . We set $X_{0}=1 / G^{\prime}$. Then the second sum above is

$$
\ll M G^{\prime} \sum_{s \leq X_{0}} \sum_{y} \frac{1}{(u+y)^{2}+\varepsilon_{0}^{2}}\left|\sum_{X(y)-s \leq x \leq X(y)} e(\psi(x))\right|
$$

so we get

$$
\begin{aligned}
& \Sigma \ll \sum_{|v|=0}^{\infty} \frac{1}{v^{4}+1} \sum_{u \leq M / 2} \sum_{y}\left\{\frac{1}{|y+u|+\varepsilon_{0}}\left|\sum_{x \in I(y)} e(\psi(x))\right|\right. \\
& \left.+\frac{G^{\prime}}{(y+u)^{2}+\varepsilon_{0}^{2}} \sum_{s \leq X_{0}}\left|\sum_{X(y)-s \leq x \leq X(y)} e(\psi(y))\right|\right\} \\
& \ll \max _{s \leq X_{0}} \sum_{|v|=0}^{V} \frac{1}{v^{4}+1} \sum_{|u| \leq M / 2} \sum_{y} \frac{1}{|y+u|+\varepsilon_{0}^{2}}\left|\sum_{X(y)-s \leq x \leq X(y)} e(\psi(x))\right|+R^{\prime},
\end{aligned}
$$


where

$$
V=X^{1 /(4 K)} \quad \text { and } \quad R^{\prime} \ll \sum_{v=V}^{\infty} v^{-4} X\left(\log X+\varepsilon_{0}^{-2}\right) \ll X^{1-1 /(4 K)} .
$$

Let $r$ be the smallest integer such that

$$
\left|f^{(r)}(x)\right| \leq X^{2 / R-1} \text { and }\left|j h^{(r)}(x)\right| \leq X^{2 / R-1} .
$$

Obviously, $1<r<k$. To evaluate the sum in (6) we need to evaluate

$$
Y(\Delta) \equiv\left|\left\{y \in\left[G_{1}, G_{2}\right]:\|\varphi(y)\| \leq \Delta\right\}\right| \quad \text { where } \quad \varphi(y)=A \psi^{(r-1)}(X(y))
$$

and $A \leq X_{0}^{1-1 / R}$ is a fixed number. Assume that $t$ is the smallest integer such that

$$
\left|\left(A f^{(r-1)}(X(y))\right)^{(t)}\right| \leq G^{2 / T-1} \quad \text { and } \quad\left|\left(A j h^{(r-1)}(X(y))\right)^{(t)}\right| \leq G^{2 / T-1} .
$$

We take a small constant $\varepsilon>0$ and divide the set of all $y$ into $\ll \log X$ intervals with

$$
\left|\varphi^{(t)}(y)\right| \lambda_{t} \geq A\left(\left|\left(f^{(r-1)}(X(y))\right)^{(t)}\right|+\left|\left(j h^{(r-1)}(X(y))\right)^{(t)}\right|\right) X_{0}^{-\varepsilon_{1}}
$$

and at most one interval, $I$, in which the above inequality is not satisfied. The conditions of the theorem imply that if $y \in I$ then

$$
\left|\varphi^{(t+1)}(y)\right| \gg A\left|\left(f^{(r-1)}(X(y))\right)^{(t+1)}\right| G^{-\varepsilon_{1}} .
$$

Using Lemma 2 with $k=t$ and $m=0$ if $y \notin I$ and $m=0$ otherwise as above we obtain

$$
\begin{aligned}
Y(\Delta) & \leq \min _{\delta \geq \Delta} Y(\delta) \\
& \ll \min _{\delta}\left(G \delta+\sum_{j=1}^{\infty} \min \left\{\delta ; 1 /\left(\delta j^{2}\right)\right\}\left|\sum_{y} e(j \varphi(y))\right|\right) \\
& \ll G \min _{\delta}\left(\delta+\sum_{j=1}^{\infty} \min \left\{\delta ; 1 /\left(\delta j^{2}\right)\right\}\right)\left(G^{-1 / T}+\left(j \mu_{t}\right)^{1 /(T-2)}\right) \\
& \ll G \min _{\delta}\left(\delta+G^{-1 / T}+\left(\mu_{t} / \delta\right)^{1 /(T-2)}\right) \\
& \leq G\left(\Delta+G^{-1 / P}+\mu_{t}^{1 /(T-1)}\right) \\
& \ll G\left(\Delta+G^{-1 / P}\right) .
\end{aligned}
$$

To evaluate the sum in (6) we assume first that $r=2$. We divide the interval $[X(y)-s, X(y)]$ into $\ll \log X$ subintervals with $\varphi^{\prime \prime}(x) \asymp \lambda_{2}$ and consider one of them, corresponding to the largest subsum. We denote it by $S(u, v, y)$.

If $\lambda_{2} \geq X_{0}^{-4 / 3}$, we use Lemma 2 with $k=2$ and $m=0$ and obtain

$$
S(u, v, y) \ll X_{0}^{2 / 3} .
$$


If $\lambda_{2} \leq X_{0}^{-4 / 3}$ we use Lemma 3 to evaluate $S(u, v, y)$ if

$$
\left\|\psi^{\prime}(X(y))\right\| \geq C X_{0} \lambda_{2} \equiv \Delta_{0}
$$

with an appropriate $C$ or evaluate it trivially otherwise.

Note that if (8) holds then for all $x \in[X(y)-s, X(y)]$ we have

$$
\left\|\psi^{\prime}(x)\right\|=\left\|\psi^{\prime}(X(y))+O\left(\lambda_{2} X_{0}\right)\right\| \asymp\left\|\psi^{\prime}(X(y))\right\| .
$$

Summing over all $u$ and $y$ and using (7), we obtain

$$
\begin{aligned}
S(v) \equiv & \sum_{u, y} S(u, v, y) \ll\left(\log X+\varepsilon_{0}^{-2}\right) \\
& \times\left[X_{0}^{2 / 3}+\sum_{l} \min \left\{X_{0} ; 1 /\left(\Delta_{0} 2^{l}\right)\right\} Y\left(\Delta_{0} 2^{l}\right)+X_{0} Y\left(\Delta_{0}\right)\right] \log X \\
\ll & \left(\log X+\varepsilon_{0}^{-2}\right)\left(G X_{0}^{2 / 3}+X_{0} G^{1-1 / P}\right) \log ^{2} X .
\end{aligned}
$$

If $r>2$, we apply Hölder's inequality to get

$$
\begin{aligned}
S(v) \ll & \left(\sum_{u, y} \frac{1}{|y+u|+\varepsilon_{0}^{2}}\right)^{1-4 / R} \\
& \times\left(\sum_{u, y} \frac{1}{|y+u|+\varepsilon_{0}^{2}}\left|\sum_{x} e(\psi(x))\right|^{R / 4}\right)^{4 / R} \log X .
\end{aligned}
$$

Now we use H. Weyl-van der Corput inequality $r-2$ times with $Q=$ $X_{0}^{4 / R}$ to obtain

$$
\begin{aligned}
S(v) \ll\left[G\left(\log X+\varepsilon_{0}^{-2}\right)\right]^{1-4 / R}\left[\sum _ { y , u } \frac { 1 } { | y + u | + \varepsilon _ { 0 } ^ { 2 } } \left(X_{0}^{R / 4} Q^{-R / 8}\right.\right. \\
\left.\left.+X_{0}^{R / 4-1} Q^{1-R / 4} \sum_{q_{1}=1}^{Q} \ldots \sum_{q_{r-2}=1}^{Q^{R / 8}}\left|\sum_{x} e\left(A \psi_{1}(x)\right)\right|\right)\right]^{4 / R}
\end{aligned}
$$

where

$$
A=q_{1} \ldots q_{r-2} \quad \text { and } \quad \psi_{1}(x)=\int_{0}^{1} \ldots \int_{0}^{1} \psi\left(x+q_{1} t_{1}+\ldots+q_{r-2} t_{r-2}\right) d \underline{t} .
$$

Using (7) we obtain, as in the proof of (9),

$$
\begin{aligned}
S(v) \ll & \varepsilon_{0}^{-2} X Q^{-1 / 2} \log ^{2} X+\left[\varepsilon_{0}^{-2} X\right]^{1-4 / R} \log X \\
& \times\left[Q^{1-R / 4} \sum_{q_{1}, \ldots, q_{r-2}} \varepsilon_{0}^{-2}\left(X_{0}^{2 / 3} G+X_{0} G^{1-1 / P}\right)\right]^{4 / R} \\
\ll & \varepsilon_{0}^{-2} X\left[X_{0}^{-4 /(3 R)}+G^{-4 /(P R)}\right] \log ^{2} X
\end{aligned}
$$


and

$\sum_{v} S(v) \ll \varepsilon_{0}^{-2} X\left[X_{0}^{-4 /(3 R)}+G^{-4 /(P R)}\right] \log ^{2} X \ll X^{1-a /(3 K)}+X G^{-1 /(P K)}$.

To prove Theorem 2 we set $n=[g(x)]$. Let $G_{1}$ and $G_{2}$ be the minimum and maximum of $g(x)$ on $[X, 2 X]$ and $G^{\prime}=g^{\prime}(X)$. Using Lemma 4, we obtain

$$
S \ll\left(\sum_{x, n} 1\right)\left(\lambda_{k}^{1 /(K-2)}+\left(G^{\prime}\right)^{-4 /(3 K)}+G^{-2 / K}+\mu_{j}^{4 /(K J+K-8)}\right) .
$$

Since $\sum_{x, n} 1 \ll X$ and $G^{\prime}=g_{1}(X) / X \ggg X^{-a}$, this completes the proof.

\section{References}

[1] D. Berend, M. Boshernitzan and G. Kolesnik, Distribution modulo 1 of some oscillating sequences, III, to be published.

[2] D. Berend and G. Kolesnik, Distribution modulo 1 of some oscillating sequences, Israel J. Math. (2) 71 (1990), 161-179.

[3] R. G. Rieger, On the integer part function and uniform distribution mod one, J. Number Theory 65 (1997), 74-86.

[4] W. G. Zhai, On the integer part function and uniform distribution modulo one, submitted for publication.

Department of Mathematics

California State University at Los Angeles

Los Angeles, CA 90032, U.S.A.

E-mail: gkolesn@exchange.calstatela.edu

Received on 28.2.2001

and in revised form on 5.12.2001 